be contributors to these tragic events happening. Cases and controls were selected from New Zealand residential properties where child injury had occurred. Analyses were carried out on the features of 88 case properties and 181 control properties. Results showed risk of injury was increased by: a driveway length of greater than 12 meters (OR 1.8, 95\% CI 1.1 to 3.0 ), exiting the driveway onto a local road (OR 5.5, 95\% CI 2.7 to 11.2 ) and the driveway exiting onto a cul de sac (OR 2.3, 95\% CI 1.4 to 3.9; when more parking areas were on the property (requiring the use of the driveway to access) (OR 3.0 $95 \%$ CI 1.6 to 5.4$)$. There was a lower risk when it a walkway or path separate to the driveway was present on the property. The study demonstrates environmental features of a property can affect the likelihood of child driveway run over injury and consideration of these results by the design and building communities is urged.

\title{
0262 CAN DRIVE-WAY DESIGN CONTRIBUTE TO TRAGEDY
}

M Shepherd, P Austin, J Chambers* Correspondence: Safekids New Zealand, PO Box 26488, Epsom, Auckland 1344, New Zealand

10.1136/ip.2010.029215.262

Driveway run-overs happen when small children are unintentionally driven over and killed or injured, by slow moving vehicles manoeuvring on private land. Personal error, vehicle design and the environment (driveway and property design) are all features thought to be important. This study investigated what elements of driveway and property design might 